\title{
APERÇU COMPARATIF SUR LE RECEL SUCCESSORAL EN DROIT PRIVE FRANÇAIS

\author{
ET ROUMAIN
}

DOI:10.24193/SUBBiur.64(2019).3.1

PublishedOnline:2019-09-25

Juanita Goicovici*

Résumé : Le recel successoral, le détournement, l'appropriation ou la soustraction frauduleuse des biens présents dans le patrimoine successoral représente une pratique assez fréquente, au défi de la réglementation prohibitive du Code civil français et roumain. Cette étude aura pour objectif d'analyser l'acceptation forcée de la succession par l'héritier dont le comportement dolosif s'est manifesté. La première condition sera celle de la qualité de successible de l'auteur du recel, au moment du détournement des biens successoraux. La seconde condition indispensable pour retenir l'existence du recel est celle de l'élément intentionnel, c'est-à-dire élément de l'intention du successible de nuire aux intérêts de ses copartageants ou des réservataires. La troisième condition est celle d'élément matériel du recel, visant les actifs patrimoniaux détournés ou distrais par l'acte frauduleux du successible. Finalement, la quatrième exigence formelle est celle de l'existence d'un acte frauduleux ou d'une action de distraction des biens de la masse successorale; il peut également s'agir de l'omission de déclarer l'existence d'un testament ou d'une libéralité rapportable ou réductible.

* Lectrice, docteur en droit, Faculté de Droit de l'Université Babeş-Bolyai de Cluj-Napoca. Email: juanitag@law.ubbcluj.ro, jgoicovici@yahoo.com. 
Mots-clefs : succession, comportement fautif, recel successoral, sanctions, successible, droit comparé.

\author{
COMPARATIVE PERSPECTIVES ON THE \\ HEIR'S CONCEALMENT OF ASSETS UNDER \\ THE FRENCH AND THE ROMANIAN \\ PRIVATE LAW
}

\begin{abstract}
Committing fraud by concealing assets or some other act or omission and misappropriation of assets represent rather frequent practices, despite the prohibitory regulation under the French and the Romanian Civil Code. We conducted an analysis on the forced acceptance of the heir the fraudulent conduct of which has been the cause of the inheritable mass imbalance. The article encompasses an analysis of the main legal exigencies for the existence of the assets concealment. The first condition refers to the quality of potential inheritor of the author of the fraudulent conduct, at the moment when the goods were swiped. The second indispensable condition refers to the intentional element or the voluntary, subjective aspect of the fraudulent behavior. The latter would cause a patrimonial loss for the other heirs, which is also required for this type of sanctioning. The third condition which follows under the scope of this analyze is that referring to the objective element of the inheritance concealment. This is materialized in the fraudulent and non-transparent managing of the assets. The article mentions the intricacies of the various types of concealment that a personal representative or potential heir can be accused of concealing assets. This type of conduct would include failure to disclose or attempting to underestimate the value of inheritance assets.
\end{abstract}

Keywords: inheritance, fraudulent conduct, concealing of assets, sanctions, potential heir, comparative law. 


\section{Observations introductives sur la notion de recel successoral ${ }^{1}$}

La notion de recel successoral est couverte d'un voile mystérieux dont la présence pourrait être regrettée (seulement) par le juriste contemporain. À la lecture des articles insérés dans le Code civil roumain, on observe que le concept de recel successoral est suffisamment permissif englobant tous actes ayant pour but de tromper les copartageants successoraux en ce qui concerne l'étendue ou l'importance de la communauté patrimoniale successorale, ainsi que toute manœuvre frauduleuse commise par un successible en vue de fragiliser ou de pulvériser l'égalité ou l'équilibre du partage successoral et partant, toute manœuvre ou tout acte de nature à causer un préjudice patrimonial aux cohéritiers. Le siège de la matière est gouverné par l'article 778 du Code civil français² et par l'article 1119 du Code

\footnotetext{
${ }^{1}$ Notons que les faits d'appropriation frauduleuse de biens successoraux qui peuvent être constitutifs de recel successoral donnant lieu à la sanction civile qui y correspond, ont le potentiel de constituer également une infraction pénale donnant lieu à des poursuites pénales en termes du Code pénal roumain.

${ }^{2}$ En droit privé français, la notion du recel successoral apparaît pour la première fois dans un arrêt de la Cour de cassation du 15 avril 1890. Celle-ci contient une définition précisant que constitue un recel successoral les manœuvres dolosives des héritiers, ainsi que la fraude commise sciemment par un héritier potentiel. Celui-ci poursuits l'objectif de rompre l'égalité ou l'équilibre du partage successoral, quels que soient les moyens employés pour y parvenir. Voir, pour la réglementation contemporaine du recel en droit français: P. MOREAU, Libéralités et successions, Anthemis, Paris, 2019, p. 187-189; A.-M. LEROYER, Droit des successions, Dalloz, Paris, 2019, p. 332. Pour le segment roumain de la réglementation en matière d'acceptation forcée de la succession par héritiers coupables, voir I. NicolAE, " Punctul de întâlnire între acceptarea forțată a moștenirii și nedemnitatea succesorală », Revista Universul juridic nr. 2/2017,http://revista.universuljuridic.ro/punctul-de-intalnireintre-acceptarea-fortata-mostenirii-si-nedemnitatea-judiciara/, adresse web consultée en 04.06.2019.
} 
civil roumain. On doit noter que l'accent est placé sur l'idée d'acceptation forcée de la succession par l'héritier dont le comportement dolosif s'est manifesté. Ces articles disposent respectivement comme suit :

Conformément aux dispositions de l'article $778 \mathrm{du}$ Code civil français : "Sans préjudice de dommages et intérêts, l'héritier qui a recelé des biens ou des droits d'une succession ou dissimulé l'existence d'un cohéritier est réputé accepter purement et simplement la succession, nonobstant toute renonciation ou acceptation à concurrence de l'actif net, sans pouvoir ne prétendre à aucune part dans les biens ou les droits détournés ou recelés. Les droits revenant à l'héritier dissimulé et qui ont où auraient pu augmenter ceux de l'auteur de la dissimulation sont réputés avoir été recelés par ce dernier ». On doit préciser que, lorsque le recel a porté sur une donation rapportable ou réductible, les systèmes successoraux, français et roumain prévoient que l'héritier doit le rapport ou la réduction de cette donation sans pouvoir y prétendre à aucune part.

Quels sont les points divergents, ainsi que les similitudes des deux réglementations? Les deux systèmes juridiques de droit privé ont en commun le fait d'exiger que l'héritier receleur soit tenu de rendre tous les fruits et revenus produits par les biens recelés dont il a eu la jouissance depuis l'ouverture de la succession. Mentionnons que, selon l'acception usuelle du recel successoral, il peut être caractérisé comme une intention frauduleuse d'un successible ayant la volonté dolosive de réduire ou changer la part d'héritage due à un autre cohéritier en déclenchant un déséquilibre 
patrimonial en plan du partage et de la liquidation successorale. La principale caractéristique est celle que le recel successoral peut être caractérisé par la dissimulation ${ }^{3}$ de certains biens ou d'une libéralité 4 .

On a abordé également, dans cette étude, le problème des différentes sanctions applicable en cas de recel successoral, en droit privé français et roumain. Notamment les questions de la preuve du caractère dolosive5, intentionnel $^{6}$ des actes ou des machinations ${ }^{7}$ du successible peuvent représenter les pillons de la réglementation du recel en droit positif.

2 L'acceptation forcée de la succession ou le cheval troyen de la réglementation légale?

Serrait l'acceptation forcée de la succession une sanction efficace du comportement fautif du successible ? Soulignons, d'abord, que les héritiers qui auraient diverti ou recelé des effets d'une succession, également en droit privé français et en droit successoral roumain, sont considérés comme déchus ${ }^{8}$ de la faculté d’y renoncer ; par ricochet, ils demeurent héritiers purs

\footnotetext{
3 A.-M. LEROYER, op. cit., p. 317.

4 Ibidem.

5 Idem, p. 319.

${ }^{6}$ Idem, p. 323.

7 Ibidem.

${ }^{8}$ Idem, p. 341.
} 
et simples ${ }^{9}$, nonobstant leur renonciation ${ }^{10}$, sans pouvoir prétendre aucune part $^{11}$ dans les objets divertis ou recelés, en intervenant ainsi une sanction pécuniaire $^{12}$ ou patrimoniale de leur comportement fautif (dolosive). Nonobstant, l'héritier qui s'est rendu coupable de receler ou qui a omis, sciemment et de mauvaise foi, de comprendre dans l'inventaire, des effets de la succession, est déchu du bénéfice d'inventaire, en termes de l'article 778 dernier paragraphe du Code civil français. L'effet reste indifférente, en termes de la réglementation du Code civil roumain, car en droit roumain, les successeurs reçoivent le patrimoine successoral en payant le pasif successoral intra vires hereditatis.

Parallèlement, la réglementation du recel en droit successoral roumain, en termes de l'article 1119 du Code civil roumain en vigueur à partir du $1^{\text {er }}$ octobre 2011, le successible qui, manifestant sa mauvaise foi, soustrait ou divertit certains biens présents dans la masse successorale ou divertit une donation rapportable ou réductible est réputé d'avoir accepté sa vocation successorale. Mentionnons que cet effet se produit même en présence d'une répudiation expresse antérieurement faite. Similairement aux dispositions de l'article 778, thèse seconde du Code civil français, le successible auteur du recel est prohibé de prétendre aucune part dans les objets divertis ou recelés de la masse successorale. La thèse seconde de l'article cité prévoit que l'héritier receleur soit tenu de rendre tous les fruits

\footnotetext{
${ }_{9}$ Idem, p. 342.

${ }^{10}$ Ibidem.

${ }^{11}$ Idem, p. 346.

12 Ibidem.
} 
et revenus produits par les biens recelés dont il a eu la jouissance depuis l'ouverture de la succession et qu'il sera tenu de couvrir sa part du passive successoral en utiliser les biens de son patrimoine, proportionnellement à sa part de l'actif successoral brut, pas nette.

Grace aux remarques faites dans la littérature spécialisée, on peut remarquer, pour le dispositif normatif français, qu'il serrait inexact de parler au sens strict d'une rupture ${ }^{13}$ de l'égalité de partage successoral ${ }^{14}$ consécutive au recel 15 commis par le successible, puisque le partage successoral n'est pas toujours égalitaire ${ }^{16}$, sans mentionner d'ailleurs que les lots peuvent avoir été fixés de manière inégale ${ }^{17}$ par le testament rédigé par le défunt ${ }^{18}$. De surcroît, il est également possible qu'un héritier réservataire ait été réduit à sa réserve par le défunt dans les limites des dispositions légales impératives sur la réserve successorale et qu'exhérédation partielle soit intervenue.

Nous soulignons aussi que, au défi des intérêts successoraux d'autres héritiers copartageants, l'acte de divertissent patrimonial accompli ou le silence gardé sur l'existence d'un testament, par exemple ou sur l'existence d'une libéralité, soit-elle rapportable ou réductible est commis avec

\footnotetext{
13 P. MOREAU, OP. CIT., P. 177.

14 Ibidem.

15 Idem, p. 181-182.

${ }^{16}$ Ibidem.

17 Ibidem.

${ }^{18}$ Idem, p. 194.
} 
l'intention frauduleuse ${ }^{19}$ d'augmenter indûment les droits du successible sur des effets successoraux ${ }^{20}$. Autrement exprimée, l'idée centrale est celle d'un comportement fautif, au préjudice de ses cohéritiers et d'ainsi porter atteint à la loyauté due aux copartageants, c'est-à-dire porter atteinte à la transparence des règles qui régissent le partage successoral, en droit privé roumain ${ }^{21}$, ainsi qu'en droit privé français ${ }^{22}$.

3 Quelles sont les exigences fondamentales et les conditions supplémentaires pour constater le recel successoral en droit prive français et roumain?

Es-que on peut identifier des conditions supplémentaires pour l'admission de l'existence du recel ? D'abord, on doit mentionner que le recel successoral ne peut être invoqué qu'à l'égard d'une personne ayant la qualité de successible. Per a contrario, la personne devenue étrangère à la

\footnotetext{
${ }^{19}$ La question se pose de savoir s'il a existé la bonne foi du successible et de vérifier s’il a omis d'évoquer une donation ou un élément de l'actif successoral ou s'il ne l'a pas déclaré par crainte de la réduction.

20 Notons que l'acte constitutif de recel successoral, c'est-à-dire l'action ou l'omission coupable du successible, doit porter sur des effets d'une succession ouverte.

${ }^{21}$ Voir D. CHIRICĂ, Tratat de drept civil. Succesiunile și liberalitățile, ediția a II-a revizuită, Hamangiu, București, 2017, p. 368-372; J. Kocsis, P. VAsILescu, Drept civil. Succesiuni, Hamangiu, București, 2016, p. 281-283; Al. BACACI, G. CoMĂNIȚĂ, Drept civil. Succesiunile, Universul juridic, București, 2015, p. 211-214.

22 Voir C. Peres, C. Vernieres, Droit des successions, Presses Universitaires de France, 2018, p. 226.-227.
} 
succession qui a renoncé23 à celle-ci ou qui a laissé passer le droit d'opter dans un intervalle légal impératif ${ }^{24}$, perd quant à elle-même l'ensemble de ses droits testamentaires ou fondés sur la succession $a b$ intestat dans les actifs de la succession. De plus, l'auteur du détournement sera condamnable ${ }^{25}$, le cas échéant, pour comportement fautif si s'approprie l'un d'entre ces actifs patrimoniaux ${ }^{26}$. Dans notre opinion, la même conclusion reste valable pour un héritier en ordre subséquent qui s'approprie des actifs successoraux auxquels d'autres que lui sont appelés conformément aux dispositions de la convention de partage volontaire. En revanche, la question se pose de savoir si, en droit roumain des successions, les successibles en ordre subséquent sont tenus responsables pour le divertissement ou sur base de l'article 1119 du Code civil (roumain) pour le recel successoral, lorsque les successeurs plus proches ont été écartés de leur vocation successorale par

23 Sont réputés purement conservatoires, sans aucun impact sur l'acceptation de la vocation successorale, des actes impliquant: le paiement des frais funéraires et de dernière maladie, des impôts dus par le défunt, des loyers et autres dettes successorales dont le règlement est urgent ; le recouvrement des fruits et revenus des biens successoraux ou la vente des biens périssables, à charge de justifier que les fonds ont été employés à éteindre les dettes visées ou ont été déposés chez un notaire ou consignés. On peut aussi nominaliser comme être conservatoire l'acte du successible, destiné à éviter l'aggravation du passif successoral.

24 Rappelons que les actes purement conservatoires constitutifs d'acceptation tacite de la vocation sont destinés à maintenir le patrimoine successoral en bon état (par exemple, réparation d'un bien, inscription d'hypothèque garantissant le payement d'une créance successorale). Le recouvrement des fruits et revenus des produits par les biens de la succession si ceux-ci sont affectés au paiement des dettes de succession représente aussi un acte conservatoire, ainsi que, plus généralement, tout acte destiné à éviter l'aggravation du passif successoral (dénonciation d'un bail du défunt, action en défense dans une action contre le défunt). Voir C. Peres, C. Vernieres, op. cit., p. 238.

25 P. MOREAU, op. cit., p. 319.

${ }^{26}$ Ibidem. 
leur indignité, leur exhérédation ou par leur renonciation expresse aux droits successoraux.

Une première condition serait donc celle de la qualité de successible en rang prioritaire ${ }^{27}$ ou ayant une vocation subséquente ${ }^{28}$ s'agissant de la distraction ${ }^{29}$ des biens donnés au défi des droits des réservataires ${ }^{30}$. On doit observer également que l'article 1119 du Code civil roumain vise les héritiers légaux comme auteurs du recel, parmi lesquels figure toujours le conjoint survivant, même lorsqu'il/elle n'hérite de la succession qu'en usufruit. En droit français des successions, le champ d'application matériel ou substantiel de cette norme juridique est cependant étendu aux légataires universels ou des quotités de la succession, et aux institués contractuels universels ou à titre universel.

Une distinction importante à faire est que si la manœuvre distractive des biens émane d'un légataire à titre particulier ou d'un donataire dont la donation reçue serait soumise à la réduction fondée sur la sollicitation expresse des réservataires, celle-ci n'est pas constitutive d'un recel successoral. En effet, on doit souligner que le légataire à titre particulier ou le donataire ne peuvent pas nuire par leur comportement dolosif à l'égalité ou a l'équilibre du partage successoral, ce qui signifie qu'il manquera une des conditions fondamentales pour l'existence du recel successoral, en

\footnotetext{
27 C. Peres, C. Vernieres, op. cit., p. 237.

28 Ibidem.

29 Idem, p. 238.

${ }^{30}$ Idem, p. 239.
} 
termes de l'article 778 du Code civil français ${ }^{31}$ et, respectivement de l'article 1119 du Code civil roumain.

La seconde condition indispensable pour retenir l'existence du recel est celle de l'élément intentionnel, c'est-à-dire élément de l'intention du successible de nuire aux intérêts de ses copartageants ou des réservataires. La tierce condition est celle d'un élément matériel du recel, visant les actifs patrimoniaux détournés ou distrais par l'acte frauduleux du successible ${ }^{32}$. Finalement, la quatrième exigence formelle est celle de l'existence d'un acte frauduleux (a) ou d'une action de distraction des biens de la masse successorale (b) ou d'omission de déclarer l'existence (c) d'un testament ou d'une libéralité rapportable ou réductible.

Nous considérons que la notion de divertissement implique un acte d'appréhension matérielle de biens successoraux, à l'opposition de commission successorale, caractérisée par l'intention de cacher l'existence des legs 33 ou l'existence d'une donation excédentaire. Le faux commis dans les actes successoraux 34 ou l'usage de faux actes 35 qui attestent faussement la qualité de successible 36 en termes de la vocation légale ou testamentaire37,

\footnotetext{
${ }^{31}$ Idem, p. 241. Voir aussi J. Maury, Successions et libéralités, LexisNexis, Paris, 2016, p. 233-236. Il pourrait être futile d'observer qu'en cas de faute commune, c'est-à-dire lorsque plusieurs cohéritiers ou plusieurs successibles participent au fait dommageable constituant le délit civil de recel, ceux-ci seront condamnés solidairement à couvrir le dommage et deviendront des acceptants forcés de leur vocation successorale individuelle.

32 Voir Al. BACACI, G. COMĂNIȚĂ, op. cit. p. 263.

33 Idem, p. 264.

34 J. MAURY, op. cit., p. 351.

35 Ibidem.

${ }^{36}$ Ibidem.
} 
ainsi que la fausse qualité de légataire ${ }^{38}$ sont aussi englobées dans le délit civil de la distraction frauduleuse des biens de la masse successorale39.

4 Les actes commission constitutifs du recel successoral en droit civil français et roumain : quelle physionomie ?

D'une perspective positiviste, le concept juridique de recel successoral, en termes de l'article 1119 du Code civil roumain, vise tant les biens immeubles, que les meubles corporels ou incorporels. L'analyse a deconspiré des valences similaires du concept de recel par l'interprétation de l'article 778 du Code civil français, bien qu'il s'agisse d'une situation moins courante, dans le cas d'une donation déguisée, par exemple si le donateur et le donataire se mettent d'accord sur un prix mais que celui-ci n'est jamais payé. Similairement, il peut s'agir des situations de recel quand au décès du donataire, le donateur tait cette donation, ces actes pourront être constitutifs de recel successoral ${ }^{40}$. Une autre hypothèse, non visée expressément par le Code civil roumain, concerne le recel d'hériter ; mentionnons que le simple fait de cacher l'existence d'un héritier pourrait, le cas échéant, être

${ }^{37}$ Idem, p. 353.

${ }^{8} 8$ Ibidem.

${ }^{39}$ Quant à l'élément matériel, puisque la doctrine semble établir une distinction entre le divertissement et le recel au sens strict, ces notions font l'objet d'éclaircissements ; voir D. CHIRICĂ, op. cit., p. 370-372.

40 Ibidem. 
constitutif de recel successoral, au défi du silence gardé sur ce sujet par les rédacteurs du Code civil roumain.

Si on compare cette mention avec celle rendue par l'article $778 \mathrm{du}$ Code civil français, on constate que cette dernière réglementation ${ }^{41}$ fait preuve d'une exigence accrue. En effet, quant au recel successoral commis par omission 42 , il peut être caractérisé comme une intention frauduleuse 43 d'un successible ayant la volonté dolosive de réduire 44 ou changer45 la part d'héritage due à un autre cohéritier en déclenchant un déséquilibre patrimonial 46 en plan du partage 47 et de la liquidation successorale ${ }^{48}$.

Pour élucider le parcours évolutif du concept de recel dans ces deux systèmes de droit civil, on doit mentionner également qu'il nous semble décisif, sinon primordial, de prendre en considération la mauvaise foi, soit la bonne foi du successible et de vérifier si le successible a omis d'évoquer une donation ou un actif patrimonial de la masse successorale parce qu'il semblait étranger au débat faisant partie de la catégorie des libéralités exceptées du rapport ou de la réduction 49 ou s'il ne l'a pas déclaré due à

${ }^{41}$ Voir C. Peres, C. Vernieres, op. cit., p. 227-228.

42 Idem, p. 231.

43 Ibidem.

44 Ibidem.

45 Idem, p. 238.

46 Ibidem.

47 Ibidem.

${ }^{48}$ Ibidem.

49 Ibidem. 
l'intention d'agir ${ }^{50}$ dans une manière contraire à la transparence ${ }^{11}$ et à la loyauté qui doivent régner entre les copartageants successoraux ${ }^{52}$.

Il convient cependant de préciser que la soustraction des biens successoraux par le successible auteur du recel n'est pas incompatible avec la remise préalable de la seule détention du bien ${ }^{53}$.

\section{Autres manœuvres frauduleux du successible.}

Quant aux manœuvres illicites du successible, notons qu'il peut s'agir aussi bien d'un faux matériel que d'un faux intellectuel commis par le successible. La dichotomie du faux matériel et du faux intellectuel en constitue la base des discussions pour envisager les valences et les facettes complexes du délit civil de recel. De plus, quant à l'énumération doctrinaire

\section{Idem, p. 229.}

${ }^{51}$ Il peut s'agir de prélever des effets de la succession dans un coffre en banque ou également de la confection et l'utilisation d'un faux testament (par le successible) en vue de s'approprier des biens successoraux. On pourrait nominaliser aussi les situations qui impliquent opérations de solder un compte du défunt après en avoir transféré les actifs sur son propre compte bancaire, ainsi que les manœuvres effectuées par un successible afin de faire croire à une donation de titres de la part du défunt alors qu'il ne disposait que d'un mandat d'administration des biens.

$5^{2}$ En effet, on doit souligner que les erreurs de fait ou de droit, commises involontairement par l'héritier, ne peuvent pas être considérées comme des manœuvres frauduleuses constitutives du recel au sens de l'article 1119 du Code civil roumain ; il y a aussi l'hypothèse du comportement involontaire à analyser. En termes de la vision positiviste sur la notion de recel, lorsque le successible agit de bonne foi et involontairement commet une faute, qu'il agit par négligence ou sans la conscience nécessaire et suffisante de ses actes, les dernières sont exemptées de l'incrimination.

53 Voir Al. BACACI, G. COMĂNIȚĂ, op. cit. p.254. 
des modes légaux couverts par l'article 1119 du Code civil roumain, ceux-ci ont fait l'objet d'une interprétation très large par la doctrine, en citant notamment la fausse signature placée par le successible sur un projet de testament élaboré par le de cujus, ainsi que la contrefaçon ou l'altération d'écriture, la fabrication de faux dispositions testamentaires englobant des legs fictifs, obligations ou décharges, les fausses déclarations sur l'absence de certains biens dans la masse successorale, l'addition de clauses au testament rédigé par le défunt, ainsi que l'omission de déclarer l'existence d'un testament par le successible auteur du recel successoral54.

Par l'usage de la pareille des concepts de la bonne foi et de la mauvaise foi du successible, nous soulignons que l'intention frauduleuse de l'auteur du recel représente le dessein subjectif ou l'intention de se procurer à soi-même un avantage patrimonial illicite au détriment de ses copartageants successoraux 55 .

On peut englober dans cette catégorie le cas de l'héritier qui, sciemment et de mauvaise foi, par un fait positif ou une dissimulation, a tenté d'accroître indûment ses droits dans la succession, ainsi que le cas de l'héritier qui, sciemment et de mauvaise foi, se prévaut d'un acte inexact ou a dissimulé l'existence d'un cohéritier, en vue augmenter frauduleusement ses droits successoraux.

\footnotetext{
54 Pour les actes compris dans la sphère d'application de l'article 778 du Code civil français, voir J. MAURY, op. cit., p. 336.

55 Les personnes concernées sont toutes celles appelées à se partager la succession, à savoir les héritiers légaux et le conjoint survivant, les légataires universels, aussi que les légataires à titre universel, à qui s'ajoutent les successeurs irréguliers et même les bénéficiaires d'une institution contractuelle.
} 
La confection d'un faux testament doit aussi être nominalisée comme constitutive du recel successoral en vue de l'article 1119 du Code civil roumain, en accentuant l'élément intentionnel ou la fraude aux droits des autres héritiers. Bref, il s'agit d'une volonté de tromper sciemment, de fausser en conscience des opérations de partage, de tronquer son égalité, bien que d'affecter l'équilibre de celui-ci. On peut également envisager que nous sommes en présence de la mauvaise foi, du mensonge nullement assimilable à la simple erreur. Nonobstant, notamment en droit privé français ${ }^{5}$, l'absence de repentir de l'héritier est essentielle pour l'incrimination du recel successoral57, car le repentir du successible en matière de recel constitué suppose une restitution spontanée et logiquement antérieure aux poursuites déclenchées contre l'auteur du recel.

\section{Quelles sont les sanctions applicables en cas de recel successoral ?}

Quelles sanctions sont applicables en vue de punir le comportement illicite du successible auteur du recel ? L'article 778 du Code civil français dispose que : «Sans préjudice de dommages et intérêts, l'héritier qui a recelé des biens ou des droits d'une succession ou dissimulé l'existence d'un cohéritier est réputée accepter purement et simplement la succession,

${ }^{6} 6$ Voir A.-M. LEROYER, op. cit., p. 366.

57Ibidem. 
nonobstant toute renonciation ou acceptation ${ }^{8}$ à concurrence de l'actif net, sans pouvoir ne prétendre à aucune part dans les biens ou les droits détournés ou recelés. Les droits revenant à l'héritier dissimulé et qui ont où auraient pu augmenter ceux de l'auteur de la dissimulation ${ }^{59}$ sont réputés avoir été recelés par ce dernier ». Il convient de souligner l'inefficacité subséquente au recel, de tous actes de répudiation expresse de sa vocation successorale légale ou testamentaire, ainsi que l'effet extinctif des droits de l'auteur du recel sur l'actif successoral net qui lui reviendra en cas contraire, c'est-à-dire en l'absence du comportement fautif constitutif de recel successoral.

Notons qu'à notre avis, en ce qui concerne l'article 1119 du Code civil roumain, s'agissant des libéralités ou donations, bien qu'il ne soit pas toujours facile de déterminer à l'avance si celles-ci excèdent ou non la quotité disponible, il paraît être excessif de juger que l'héritier gratifié est tenu de révéler toutes les libéralités qui lui ont été faites, car elles

${ }^{5}$ En droit successoral roumain, similairement au droit civil français, l'acceptation tacite ou implicite de la vocation successorale peut revêtir la forme de la cession des droits successifs par un héritier à un cohéritier ou à un tiers. Celle-ci implique une acceptation de la vocation successorale légale ou testamentaire du cedens, car pour céder ses droits successoraux, il faut au préalable avoir accepté la succession. Il y a acceptation tacite en présence d'une renonciation gratuite des droits successifs par un héritier au profit de certains cohéritiers déterminés ou nominalisés. Dans ce dernier cas, le renonçant règle lui-même le sort de la part à laquelle il renonce. Cela signifie donc, en termes de l'acceptation tacite, que le cèdent a accepté, au préalable, sa vocation à la succession ouverte. La renonciation onéreuse au profit de certains ou de tous les cohéritiers implique néanmoins une acceptation implicite de ses droits successoraux, car le successible renonçant perçoit le prix de la renonciation ce qui revient en fait à lui attribuer sa vocation successorale. Voir D. CHIRICĂ, op. cit., p. 329.

59Il s'agit de tout acte malhonnête par lequel un héritier cherche délibérément à obtenir une part d'héritage supérieure à celle à laquelle il a droit légalement. 
constituent un élément dont on doit tenir compte dans la liquidation de la succession et qui peuvent influer sur la détermination des droits des héritiers. Comme une proposition pour une future réglementation, il parait plus juste de l'obliger à rapporter seulement les donations (qui pourront être excédentaires) faites pendant un intervalle de 5 - 7 ans qui précèdent le moment de l'ouverture de la succession.

Un aspect épatant serrait celui de l'effet exclusif du recel, ainsi que les droits revenant à l'héritier dissimulé et qui ont où auraient pu augmenter ceux de l'auteur de la dissimulation sont réputés avoir été recelés par ce dernier ${ }^{60}$.Indépendamment de la sanction légale du recel, qui répare les conséquences matérielles du recel successoral, l'article $778 \mathrm{du}$ Code civil français prévoit l'octroi de dommages-intérêts aux copartageants affectés ; par comparaison, bien que les dispositions de l'article 1119 du Code civil roumain ne mentionnent pas expressément le payement des dommages-intérêts, cet effet reste possible sur le fondement des dispositions légales générales du Code civil roumain en matière de responsabilité civile délictuelle.

60 Ce qui est intéressant de noter est que, dans l'interprétation donnée par la Cassation française dans l'application de la réglementation précédente (Cass. 1ère Civ., 4 juin 2009, pourvoi $\mathrm{n}^{\circ}$ 08-15.093), la dissimulation du capital d'une assurance vie par un héritier est constitutive d'un recel successoral ; de plus, si les primes versées par le souscripteur sont manifestement exagérées, celles-ci constituent des libéralités dont il doit être tenu compte dans la liquidation de la succession. 
7 Réverbérations punitives et perspectives civilistes sur le recel successoral

Quelles sont les réverbérations punitives de la réglementation citée ? La perte des droits sur le bien recelé représente la caractéristique majeure d'apparat punitif en matière de recel, ainsi que la perte de la faculté du successible de renoncer ou d'accepter la succession à concurrence de l'actif net. Brièvement, il s'agit de la perte ou la déchéance de l'exercice du droit potestatif de l'option successorale, car le receleur ne pourra plus refuser ou accepter une succession à concurrence de l'actif net. Ainsi, si la succession est déficitaire et la valeur du passif successoral est supérieure à celle de l'actif successoral, l'auteur du recel devra en assumer les charges en réalisant le payement.

Quant à la perte des droits sur la part des objets divertis ou recelés, cet effet ${ }^{61}$ s'ajoute à celui visant le rapport à la masse successorale, car en cas de recel de donation rapportable ou réductible, l'héritier doit le rapport ou la réduction de cette donation, sur laquelle il aura perdu ses droits. Finalement, l'obligation de restituer tous les fruits et revenus perçus sur l'objet recelé est en effet une réverbération directe de la qualité de possesseur de mauvaise foi de l'auteur du recel successoral.

Des omissions, commisses par les successibles durant la période intérimaire entre l'ouverture de la succession et la liquidation de la masse

${ }^{61}$ Il sanctionne les héritiers rendus auteurs, complices ou receleurs de détournements de biens, d'actifs ou de droits successoraux. 
successorale, peuvent être fautives, mais ne pas être constitutives de recel successoral. La loi roumaine dispose ainsi que la simple omission d'un bien indivis donne lieu à un partage complémentaire portant sur ce bien omis. Le recel de succession fautif suppose véritablement une mauvaise foi ou une intention frauduleuse de l'auteur de la dissimulation. En effet, les juges ne sanctionnent le recel de succession que si la preuve est rapportée, par ceux qui l'invoquent, que son auteur a eu l'intention de tromper ses cohéritiers.

\section{Notes conclusives.}

Pour élucider le parcours évolutif du concept de recel dans ces deux systèmes de droit civil, en termes de l'article 778 du Code civil français et de l'article 1119 du Code civil roumain, on a mentionné dans les paragraphes précédents qu'il nous semble décisif, sinon primordial de prendre en considération la mauvaise foi, soit la bonne foi du successible. Il s'agit, en termes de la probation processuelle, de vérifier si le successible a omis d'évoquer une donation ou un actif patrimonial de la masse successorale parce qu'il semblait étranger au débat faisant partie de la catégorie des libéralités exceptées du rapport ou de la réduction ${ }^{62}$ ou s'il ne l'a pas déclaré due à l'intention d'agir dans une manière contraire a la transparence et à la loyauté qui doive régner entre les copartageants successoraux.

Nonobstant, l'idée que la qualité d'héritier de celui qui a commis le recel est primordiale, ou du moins du complice du recel reste fondamentale.

62 Voir C. PERES, C. VERnieres, op. cit., p. 227. 
En effet, nous avons souligné dans les paragraphes précédents que, si le défunt est à l'initiative du recel et que l'héritier est un complice, le recel successoral sera tout de même reconnu. Seulement les personnes qui ont une vocation successorale effective peuvent être accusées d'avoir commis le recel successoral. Ainsi, les légataires à titre particulier ou les donataires dont la libéralité reçue n'est pas assujettie au rapport ou à la réduction en vue de protéger la réserve, c'est-à-dire les bénéficiaires d'une donation non excédentaire du défunt, ne sont pas susceptibles d'être poursuivi pour recel successoral.

Une seconde conclusion indispensable est celle que les erreurs, de fait ou de droit, commises involontairement par héritier, ne peuvent pas être considérées comme une manœuvre frauduleuse constitutive du recel au sens de l'article 1119 du Code civil roumain, ni au sens des dispositions de l'article 778 du Code civil français; lorsque le successible agit de bonne foi et involontairement commet une faute, qu'il agit par négligence ou sans la conscience nécessaire et suffisante de ses actes, les dernières manœuvres sont exemptées de la sphère matérielle et intentionnelle du recel successoral.

Cet article met en balance les deux facettes du recel successoral ; bien que l'intention frauduleuse soit une condition nécessaire, il faut encore que celle-ci se manifeste matériellement par un divertissement d'un ou plusieurs effets patrimoniaux de la masse successorale. Une caractéristique commune aux deux systèmes juridiques qui font l'objet de la comparaison est celle que l'auteur du recel successoral doit avoir l'intention de s'approprier le bien remis par le défunt ou de dépouiller le cohéritier d’un bien qui lui appartient. 
Pour en conclure, on est persuadée par l'idée que le successible qui s'approprie des choses de la masse successorale doit avoir agi dans le but d'en disposer en tant que propriétaire et de procurer à lui-même ou autrui un avantage illicite, en disparité avec l'obligation de transparence entre les copartageants successoraux.

En somme, sans qu'énumération soit exhaustive, on puisse nominaliser comme constitutives de recel la dissimulation d'un héritier, la dissimulation ou destruction d'un testament non enregistré du défunt, la rédaction d'un faux testament olographe à la place du défunt, la dissimulation d'argent du défunt ou d'objets de celui-ci, la dissimulation d'une donation excédentaire du vivant du défunt.

L'appréciation de l'existence du recel successoral est une question de fait $^{63}$, librement soumise à l'appréciation des juges du fond, dans les deux systèmes de droit civil qui font l'objet de cette comparaison. Le fait constitutif de recel successoral ou la dissimulation de biens successoraux doit se matérialiser nécessairement après l'ouverture de la succession, c'està-dire après le décès du défunt, avant la réalisation du partage de l'indivision post-communautaire entre les héritiers. Les droits revenant à l'héritier dissimulé et qui ont ou auraient pu augmenter ceux de l'auteur de la dissimulation sont réputés avoir été recelés par ce dernier.

Les éléments communs des réglementations portant sur le recel successoral en droit français et en droit civil roumain englobent ceux

${ }^{63}$ C'est ce que la première chambre Civile de la Cour française de cassation a rappelé le 12 juin 2014, $N^{\circ}$ pourvoi: 13-17074. 
référentiels aux sanctions du comportement dolosif. Ainsi, l'auteur du recel peut être condamné à la privation totale de ses droits sur les biens divertis ou recelés, attachée à l'obligation d’indemniser les copartageantes victimes du recel par l'octroi de dommages-intérêts, même si les autres peines civiles du recel étaient écartées en raison du repentir de son auteur (restitution volontaire des biens détournés avant le début des poursuites judiciaires). La déchéance de plein droit de la faculté d'option que la loi reconnaît aux successibles entre-vient aussi, de sorte que l'auteur du recel est réputé accepter purement et simplement la succession, nonobstant toute renonciation ou acceptation à concurrence de l'actif net et pourra avoir à assumer sur son patrimoine les dettes successorales à proportion de ses droits dans la succession à l'égard des héritiers et des créanciers de la succession.

À notre avis, la restitution doit porter sur le bien dans l'état où il se trouvait au jour de l'ouverture de la succession de façon à ce que l'héritier receleur soit censé, depuis l'ouverture de la succession, n'en avoir jamais eu la propriété des actifs patrimoniaux soustraits, en qualité de possesseur de mauvaise foi. Si le bien recelé est une somme d'argent, on apprécie que la restitution doive porter sur cette valeur pécuniaire, sauf les cas où la somme détournée a été employée par le receleur à acquérir un bien ; il a été jugé64 que la restitution doit être calculée d'après la valeur de ce bien à l'époque du partage successoral.

64 Voir la jurisprudence citée par J. MAURY, op. cit., p. 266-267. 